\title{
Model-driven Autotuning of Sparse Matrix-Vector Multiply on GPUs
}

\author{
Jee W. Choi \\ Georgia Institute of Technology, School \\ of Electrical and Computer Engineering \\ Atlanta, Georgia, USA \\ jee@ece.gatech.edu
}

\author{
Amik Singh \\ Indian Institute of Technology Roorkee, \\ Department of Electronics and Computer \\ Engineering, \\ Roorkee, India \\ amiksuec@iitr.ernet.in
}

\author{
Richard W. Vuduc \\ Georgia Institute of Technology, \\ Computational Science and Engineering \\ Division \\ Atlanta, Georgia, USA \\ richie@cc.gatech.edu
}

\begin{abstract}
We present a performance model-driven framework for automated performance tuning (autotuning) of sparse matrix-vector multiply (SpMV) on systems accelerated by graphics processing units (GPU). Our study consists of two parts.

First, we describe several carefully hand-tuned SpMV implementations for GPUs, identifying key GPU-specific performance limitations, enhancements, and tuning opportunities. These implementations, which include variants on classical blocked compressed sparse row (BCSR) and blocked ELLPACK (BELLPACK) storage formats, match or exceed state-of-the-art implementations. For instance, our best BELLPACK implementation achieves up to $29.0 \mathrm{Gflop} / \mathrm{s}$ in single-precision and $15.7 \mathrm{Gflop} / \mathrm{s}$ in doubleprecision on the NVIDIA T10P multiprocessor (C1060), enhancing prior state-of-the-art unblocked implementations (Bell and Garland, 2009) by up to $1.8 \times$ and $1.5 \times$ for single- and doubleprecision respectively.

However, achieving this level of performance requires input matrix-dependent parameter tuning. Thus, in the second part of this study, we develop a performance model that can guide tuning. Like prior autotuning models for CPUs (e.g., Im, Yelick, and Vuduc, 2004), this model requires offline measurements and run-time estimation, but more directly models the structure of multithreaded vector processors like GPUs. We show that our model can identify the implementations that achieve within $15 \%$ of those found through exhaustive search.
\end{abstract}

Categories and Subject Descriptors C.4 [Computer Systems Organization]: Performance of Systems-Modeling Techniques

General Terms Algorithms, Performance

Keywords GPU, sparse matrix-vector multiplication, performance modeling

\section{Introduction}

We consider the sparse matrix-vector multiply (SpMV) operation for platforms based on graphics processing units (GPUs). Our moti-

Permission to make digital or hard copies of all or part of this work for personal or classroom use is granted without fee provided that copies are not made or distributed for profit or commercial advantage and that copies bear this notice and the full citation on the first page. To copy otherwise, to republish, to post on servers or to redistribute to lists, requires prior specific permission and/or a fee.

PPoPP'10, January 9-14, 2010, Bangalore, India.

Copyright (C) 2010 ACM 978-1-60558-708-0/10/01 .. \$10.00 vation comes from two well-known facts: (1) To first order, streaming the matrix should dominate SpMV performance, and so SpMV is largely memory bandwidth-bound; and (2) current and emerging GPUs are bandwidth-rich computing platforms, today offering peak bandwidths an order of magnitude higher than those of conventional multicore platforms based on general-purpose CPUs. Thus, SpMV and GPUs should be a perfect match.

The challenge is that SpMV is an irregular computation that, in addition to streaming, requires many indirect and irregular memory accesses. This memory behavior contrasts starkly to that of dense linear algebra kernels, such as LU, QR and Cholesky factorizations, for which highly efficient implementations exist that achieve hundreds of Gflop/s on a single GPU [17]. An SpMV incurs the overhead of moving integer indices (meta-data) that track which nonzeros are stored, and furthermore performs many indirect loads.

Indeed, from a productivity perspective, the dense and sparse cases for matrix-vector multiply differ markedly. Without prior knowledge of NVIDIA GPUs and using only the information provided in the CUDA programming guide [1], we wrote a dense matrix-vector multiplication kernel that achieves $92 \%$ of the bandwidth measured using the bandwidthTest program provided with the CUDA SDK. ${ }^{1}$ Achieving even half the performance for SpMV required significantly more effort, including designing a suitable data structure as well as careful tuning. Our SpMV implementation contains roughly twice the number of lines of CUDA code as the dense counterpart.

Findings and contributions. This paper makes several contributions.

First, we implement the classical blocked version of CSR (BCSR) and study the effects of common GPU optimizations on the kernel. Tuning BCSR for GPUs differs markedly from an equivalent CPU implementation. From this experience, we design and implement a new sparse matrix compression format, called BELLPACK, tuned for GPUs. This format extends ELLPACK/ITPACK format [14] with (a) explicit storage of dense blocks to compress the data structure, and (b) row permutation to avoid unevenly distributed workloads. The absolute performance achieved, up to 29.0 Gflop/s in single-precision and $15.7 \mathrm{Gflop} / \mathrm{s}$ in double-precision on a single NVIDIA T10P multiprocessor-based GPU, enables improvements over the best unblocked state-of-the-art implementation by up to $1.8 \times$ and $1.5 \times$ for single and double-precision computations respectively [3].

However, BELLPACK requires careful tuning. Thus, we propose a novel and accurate performance model-driven framework

\footnotetext{
${ }^{1}$ At the time, up to over twice the performance of the cublas Sgemv kernel in the CUBLAS library.
} 
for autotuning SpMV, based on an abstract execution model of a GPU. This framework, based on the paradigm of offline benchmarking combined with a model instantiated at run-time [11], selects the correct tuning parameters with a median error relative to exhaustive search of less than $15 \%$.

Limitations. Our study is encouraging but not without limitations. First, the improvements due to BELLPACK apply only to matrices that have small dense block sub-structures; however, this class is still fairly broad as it includes matrices arising in applications based on the finite-element method. Also, using BELLPACK involves re-packing the data from its original form, such as CSR, which incurs a run-time cost. However, this cost is inherent in any autotuning scheme that considers transformations of the data structure $[11,18]$.

Our execution model of the GPU is our first attempt, and does not directly model some costs, such as thread block scheduling costs, which we believe to be small. Also, although the autotuning framework can be extended to other GPU-like multithreaded SIMD architectures, we test it in this paper on only two NVIDIA Tesla series GPUs on two different kernels. Extending this framework to other architectures may require some modifications or additions to the model. Nevertheless, we believe that, by validating on an irregular computation, we complement the most sophisticated GPU models developed to date [10].

\section{Related Research}

The literature on SpMV optimization and tuning is extensive. We review the most relevant work here, and refer the interested reader to other surveys $[7,11,19]$.

This paper follows in the spirit of the paper by Williams, et al., which evaluates SpMV for several multicore platforms, including the Sony-Toshiba-IBM Cell/Broadband Engine (STI Cell/B.E.) but excluding GPUs [21]. Bell and Garland consider several methods, including a variation of ELLPACK that differs from ours [3]. They split the storage between an ELLPACK and coordinate format to reduce its footprint, a novel variant of other previously proposed splitting methods $[9,13,20]$. At the same time, Baskaran and Bordawekar proposed a general compile- and run-time infrastructure, evaluated for SpMV [2]. They seem to achieve performance roughly comparable to Bell and Garland on similar hardware.

Bolz, et al., published the first paper in 2003 on GPU-based SpMV (plus higher-level multigrid and conjugate gradient solvers) of which we are aware [4]. They achieved a high fraction $(\sim$ 1/3) of GPU peak for structured grids. Sengupta, et al., developed more generic approaches using parallel prefix/scan primitives [16], though this implementation did not at the time outperform CPUbased codes $[8,16]$. Christen and Schenk accelerate the dense part of a sparse direct solver, whereas SpMV-centric studies implicitly target iterative solvers [5].

The row permutation employed by our proposed BELLPACK format is directly inspired by early work on vectorized SpMV, which is especially relevant to GPUs. This prior work includes jagged diagonal or clever row/column permutations combined with traditional formats $[6,12,13]$.

\section{NVIDIA CUDA and Experimental Setup}

NVIDIA's CUDA is a programming model designed for the dataparallel processing capabilities of NVIDIA GPUs. A CUDA program consists of a host program that runs on the CPU host, and a kernel program that executes on the GPU itself. The host program typically sets up the data and transfers it to and from the GPU, while the kernel program processes that data.

The CUDA model has two key components. The first is the concept of fine-grained threads, which are grouped into coarser-

\begin{tabular}{|c|c|c|}
\hline & Testbed 1 & Testbed 2 \\
\hline GPU & Tesla C1060 & Tesla C870 \\
\hline GPU Clock & $1.44 \mathrm{GHz}$ & $1.35 \mathrm{GHz}$ \\
\hline Compute Capability & 1.3 & 1.0 \\
\hline CUDA Version & 2.2 & 2.0 \\
\hline bandwidthTest & $72 \mathrm{~GB} / \mathrm{s}$ & $57 \mathrm{~GB} / \mathrm{s}$ \\
\hline
\end{tabular}

Table 1. NVIDIA GPU testbeds used in our study.

grained thread blocks. Threads within a block share a local-store and may synchronize via barriers. There is no such synchronization mechanism for threads in different thread blocks.

The second component is the memory hierarchy. The main memory in a GPU is a high-bandwidth DRAM in a shared address space. There is also a low-latency per-thread-block on-chip memory called the shared memory and a per-thread private local memory in the form of registers. There are also two types of readonly memory called constant memory and texture memory, also uniformly addressable by all threads.

The CUDA Programming Guide offers several tips for maximizing performance [1]. (1) Maximize the bandwidth from global memory using coalesced loads. (2) The latency from the on-chip shared memory is comparable to that of the register file. Therefore, shared memory should be used to store data that is shared amongst the threads and/or reused frequently. (3) Resource usage - the number of threads, amount of shared memory, and the number of registers used by a single thread block-determines multiprocessor utilization, and kernels must carefully balance usage of these resources. Other "folk" methods include loop unrolling, interleaving memory accesses and processing to hide memory latencies. Volkov and Demmel offer additional techniques, and argue that a suitable machine abstraction for GPUs is that of a multithreaded local-store vector architecture [17].

Experiments. All experiments in this paper were run on either or both of the two systems listed in Table 1, and using the sparse matrix benchmark suites in Table 2, which are used in prior work by others $[3,21]$.

\section{Hand-optimizing Baseline BCSR}

This section describes a basic blocked compressed sparse row (BCSR) implementation that extends the state-of-the-art GPU CSR implementation [3]. From this basic implementation, we identify key factors that prevent the algorithm from achieving better performance and hand-optimize the kernel accordingly.

\subsection{CSR and BCSR}

The conventional CSR format stores an $m \times n$ sparse matrix having $k$ non-zero elements using three one-dimensional arrays: the arrays val and col_ind, each of size $k$, to store the non-zeros values and column indices, respectively; and an array row_ptr of size $m+1$ to store pointers to the first element of each row in the val and col_ind arrays. In the implementation by Bell and Garland, a warp of threads is assigned to each row of the matrix, with each thread performing a multiply-accumulate on the non-zeros in the row in an interleaved manner. After all non-zeros have been processed and stored in the shared memory (local-store), the warp executes a parallel reduction to produce the final value.

In the blocked variant of CSR, BCSR, we store $r \times c$ dense subblocks of non-zeros rather than storing each non-zero individually [15], as illustrated in Figure 1. Depending on the matrix, we can in principle reduce the column index storage by up to roughly $\frac{1}{r \cdot c}$, since we need only store 1 column index per block. 


\begin{tabular}{|c|c|c|c|}
\hline Name & Dimensions & $\begin{array}{l}\text { Non- } \\
\text { zeros }\end{array}$ & Description \\
\hline Dense & $2 \mathrm{~K} \times 2 \mathrm{~K}$ & $4.0 \mathrm{M}$ & Dense \\
\hline Protein & $36 \mathrm{~K} \times 36 \mathrm{~K}$ & $4.3 \mathrm{M}$ & $\begin{array}{l}\text { Protein data } \\
\text { bank } 1 \mathrm{HY} 2 \mathrm{~S}\end{array}$ \\
\hline QCD & $49 \mathrm{~K} \times 49 \mathrm{~K}$ & $1.9 \mathrm{M}$ & $\begin{array}{c}\text { Quark } \\
\text { propagation }\end{array}$ \\
\hline Cantilever & $62 \mathrm{~K} \times 62 \mathrm{~K}$ & $4.0 \mathrm{M}$ & Cantilever \\
\hline Spheres & $83 \mathrm{~K} \times 83 \mathrm{~K}$ & $6.0 \mathrm{M}$ & $\begin{array}{l}\text { Concentric } \\
\text { spheres }\end{array}$ \\
\hline Harbor & $47 \mathrm{~K} \times 47 \mathrm{~K}$ & $2.37 \mathrm{M}$ & $\begin{array}{l}\text { 3D CFD of } \\
\text { Charleston } \\
\text { Harbor }\end{array}$ \\
\hline Ship & $141 \mathrm{~K} \times 141 \mathrm{~K}$ & $3.98 \mathrm{M}$ & Ship section \\
\hline Wind Tunnel & $218 \mathrm{~K} \times 218 \mathrm{~K}$ & $11.6 \mathrm{M}$ & $\begin{array}{l}\text { Pressurized } \\
\text { wind tunnel }\end{array}$ \\
\hline Cop & $121 \mathrm{~K} \times 121 \mathrm{k}$ & $2.6 \mathrm{M}$ & Accelerator \\
\hline Epidemiology & $526 \mathrm{~K} \times 526 \mathrm{~K}$ & $2.1 \mathrm{M}$ & $\begin{array}{l}\text { 2D Markov } \\
\text { model of } \\
\text { epidemic }\end{array}$ \\
\hline Economics & $207 \mathrm{~K} \times 207 \mathrm{~K}$ & $1.27 \mathrm{M}$ & $\begin{array}{l}\text { Macro- } \\
\text { economics } \\
\text { model }\end{array}$ \\
\hline Circuit & $171 \mathrm{~K} \times 171 \mathrm{~K}$ & $959 \mathrm{~K}$ & $\begin{array}{l}\text { Motorola } \\
\text { circuit } \\
\text { simulation }\end{array}$ \\
\hline Webbase & $1 \mathrm{M} \times 1 \mathrm{M}$ & $3.1 \mathrm{M}$ & $\begin{array}{c}\text { Web } \\
\text { connectivity }\end{array}$ \\
\hline LP & $4 \mathrm{~K} \times 1 \mathrm{M}$ & $11.3 \mathrm{M}$ & $\begin{array}{c}\text { Linear } \\
\text { programming }\end{array}$ \\
\hline
\end{tabular}

Table 2. Overview of sparse matrices used in evaluation study. See also Williams, et al. [21].
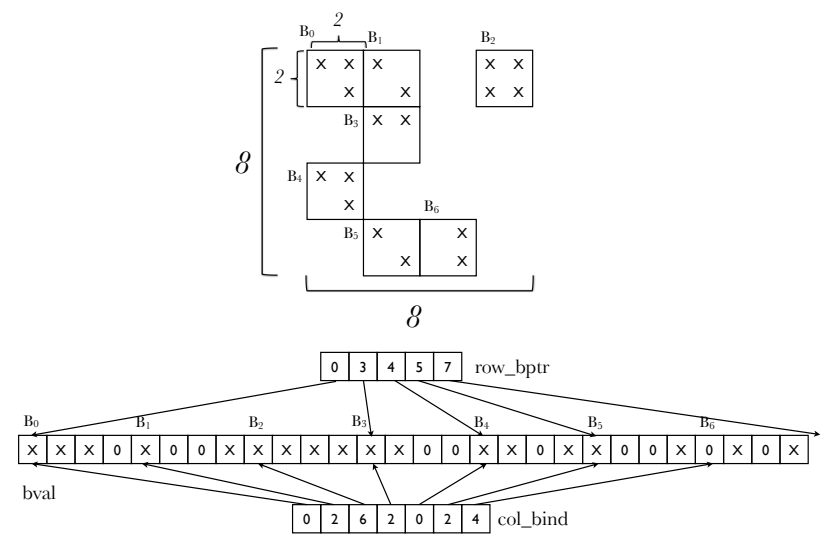

Figure 1. The BCSR compression format.

Given a matrix in CSR, we implement BCSR by statically dividing the matrix into $\left(\frac{m}{r}\right) \times\left(\frac{n}{c}\right)$ sub-blocks of size $r \times c$ each, with explicit padding of zeros as needed.

Our initial BCSR implementation begins with a straightforward adaptation of the CSR baseline of Bell and Garland [3]. In particular, we store elements of each $r \times c$ block contiguously and assign each thread to an $r \times c$ block, combining the results within a block row via parallel reduction. The pseudocode for SpMV when $r=c=2$ appears in Algorithm 1.

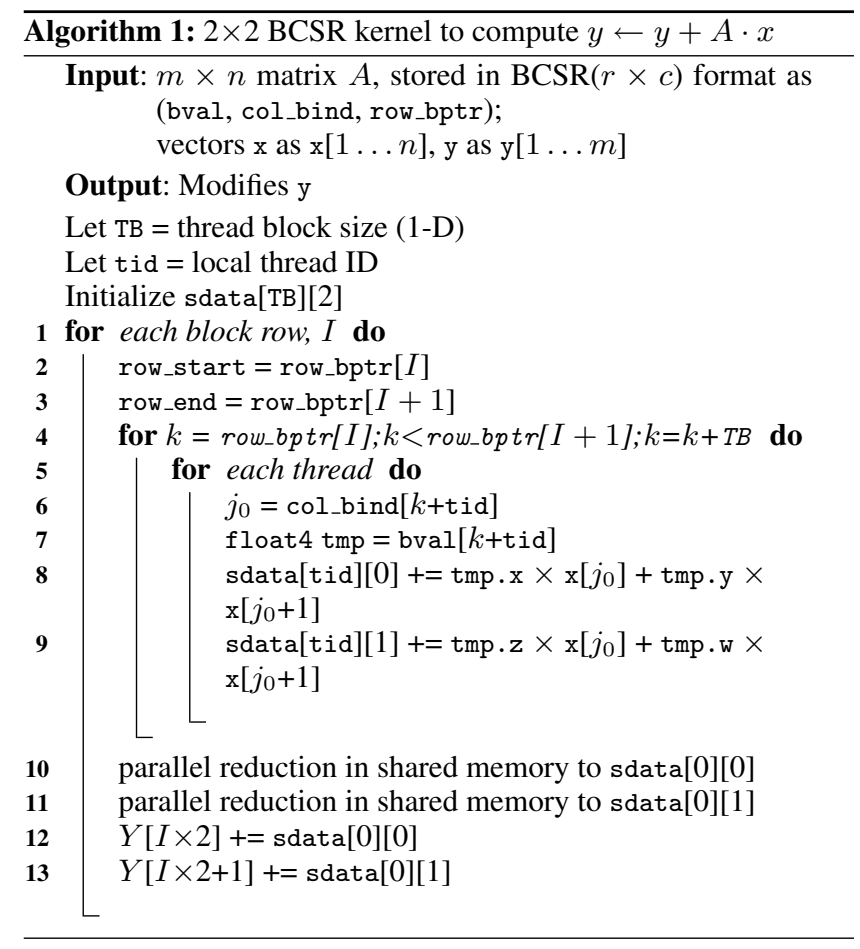

The performance of this baseline implementation is poor, owing largely to uncoalesced memory accesses. We address this issue through the transformations described below.

\subsection{Short Vector Packing}

Because each thread is assigned to a particular $r \times c$ block and each block is stored contiguously as $r \times c$ floats, threads within a warp will access data in a non-contiguous manner, leading to a deterioration in performance.

We can partially alleviate this problem by first loading data in larger granularities. In particular, we exploit CUDA's built-in shortvector data types, float2, float3, and float4, which correspond to 32-, 64-, and 128-bit vectors. When $r \times c$ is small, e.g., $2 \times 2$, $3 \times 1$, or $1 \times 4$, the entire block will fit into a single short vector, so that threads within a warp issue contiguous short-vector loads, thereby reducing the instruction count and improving bandwidth utilization.

If an $r \times c$ block requires more than 1 short vector, we will still have non-contiguous loads if we store the multiple short vectors contiguously (addressed below). For $1 \times 3,3 \times 1$, and $3 \times 3$ blocks, we find empirically that it is generally better to use float 4 storage with padded zeros, since float4 is automatically aligned [1].

\subsection{Row Alignment}

When the number of $r \times c$ blocks in any block row do not sum to multiples of the word boundary required for alignment and coalesced access, all subsequent accesses to the matrix will become uncoalesced. This problem can be easily solved by padding each row block with explicit $r \times c$ blocks containing all zeros, at the cost of extra storage and flops.

\subsection{Interleaved Memory Accesses}

When multiple short-vector words are required to store an $r \times c$ block, we can avoid non-contiguous short vector access within a warp by interleaving words from consecutive blocks. 


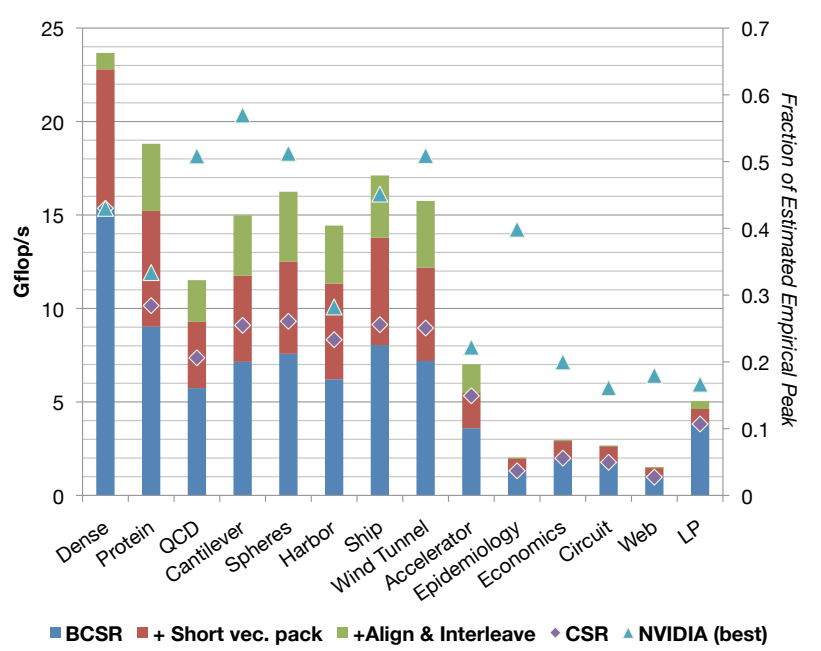

Figure 2. Single-precision performance (Gflop/s) of the best BCSR kernels for each test matrix. The estimated empirical peak (secondary y-axis on right) assumes an ideal 2 flops per 4 bytes streamed at the empirical peak bandwidth of $72 \mathrm{~GB} / \mathrm{s}$. This bandwidth is reported by the CUDA SDK bandwidthTest benchmark.

\subsection{Experimental Results}

We compare the BCSR implementations to the NVIDIA implementations [3] on the NVIDIA C1060 (T10P-based) platform in Figure 2 . The block size is tuned exhaustively up to $4 \times 4$, reporting the best case. Not surprisingly, the final BCSR implementation outperforms the baseline CSR implementation for matrices with natural dense block substructure-Protein through Accelerator, as these matrices come predominantly from simulations based on the finite-element method.

However, the NVIDIA's best implementations using other formats (e.g., the hybrid ELLPACK+coordinate (COO) scheme) still outperform our best BCSR code in several cases. Our best BCSR rarely exceeds $50 \%$ of the estimated peak for single-precision dense matrix-vector multiply. Thus, even if the application is bandwidthlimited like SpMV, simply reducing the data size is inadequatespecial optimizations are also necessary to achieve still higher performances on GPUs.

\section{Blocked ELLPACK}

Although the BCSR improves over CSR, it still falls far short of NVIDIA's best implementation (Figure 2). The major bottleneck comes from the parallel reduction step, whose performance is sensitive to the number of blocks available in a particular block row, which can be small (e.g., less than 20). Given the similarity between current GPUs and vector architectures [17], we might instead prefer a vector-friendly format such as the classical ELLPACK/ITPACK format [14]. Indeed, the best NVIDIA format is often (but not always) their hybrid ELLPACK+COO scheme [3]. Therefore, we also consider a blocked ELLPACK (BELLPACK) format that combines the advantages of the dense subblock storage of BCSR and the vector-friendly ELLPACK format.

In classical ELLPACK, we store an $m \times n$ matrix using two $m \times$ $L$ arrays, for the values and column indices, respectively, where $L$ is the maximum number of non-zeros in any row. That is, an ELLPACK matrix is stored using two $m \times L$ arrays $\mathrm{V}$ and $\mathrm{J}$ such that $\mathrm{v}[i, k]$ is the matrix entry $A(i, \mathrm{~J}[i, k])$. Rows with fewer than $L$ nonzeros are padded with zeros, which results in unnecessary storage and flops (work). However, this format suits GPUs well because it

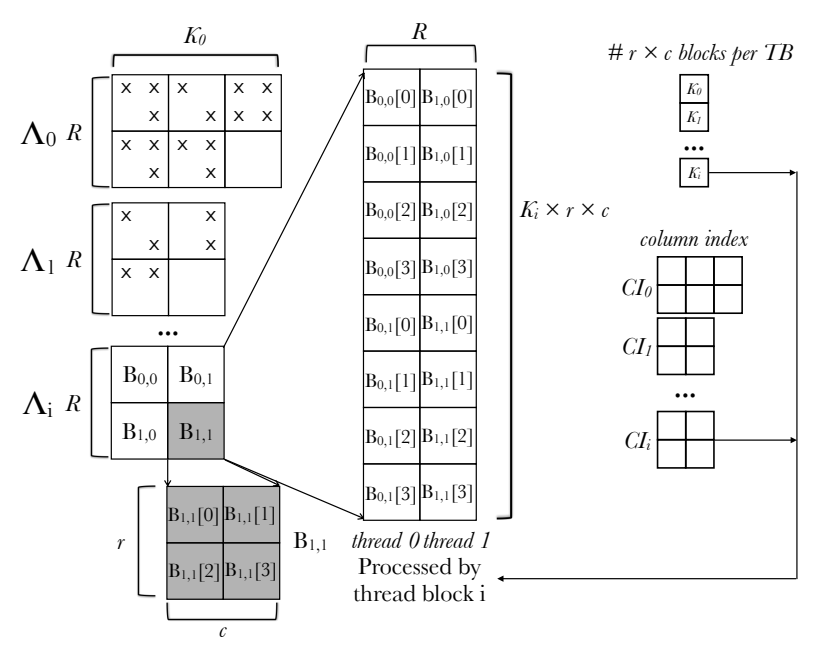

Figure 4. Storage of block rows in BELLPACK

is easy to (a) vectorize SpMV within a column $\mathrm{v}[\cdot, k]$, given indirect addressing support; (b) align the matrix data for efficient global memory transfers; and (c) do 1-D block row-based partitioning. Storing $r \times c$ dense subblocks is a straightforward adaptation, helping to reduce column index storage as with BCSR. However, ELLPACK peforms poorly when the variance in the number of nonzeros per row is high, thereby necessitating excessive zero-padding.

\subsection{Our Blocked ELLPACK Implementation}

Conceptually, we construct a BELLPACK matrix from a $m \times n$ input matrix $A$ as sketched in Figure 3(a) and described as follows.

Basic storage scheme. First, we logically reorganize $A$ into a new matrix, $A^{\prime}$, stored using $r \times c$ dense subblocks. This step helps reduce column index storage, as with BCSR. We then sort the block-rows in descending order of number of blocks per row, resulting in a new matrix $A^{\prime \prime}$. The sort step is identical to what is done for the so-called jagged diagonal format [15], and corresponds to applying a row permutation, $P_{r}$, to $A^{\prime}$, i.e., $A^{\prime \prime}=P_{r} \cdot A^{\prime}$. Finally, we partition the rows of $A^{\prime \prime}$ into $\frac{m}{R}$ non-overlapping submatrices, each of size $R \times \frac{n}{c}$. We store each submatrix in ELLPACK or $r \times c$ blocked ELLPACK format. This partitioning step helps to reduce the padding that would otherwise result in matrices with a high variance in the number of non-zeros per row, as suggested in Figure 3(b).

Block row-partition layout. To encourage coalescing, we store each $R \times \frac{n}{c}$ block row, $\Lambda_{i}$, as illustrated in Figure 4. In particular, the values of $\Lambda_{i}$ are laid out in a 2-D row-major array (middle of Figure 4). Each column contains all of the data to-be-accessed by a particular thread, laid out $r \times c$ block-by-block. The unit-stride dimension is across rows of this array, to ensure coalescing. This array is aligned and padded as necessary. A parallel structure is used to store the block column indices (right side of Figure 4).

The pseudocode for a $2 \times 2$ kernel appears in Algorithm 2 . Blocking and processing the data as described above leads to an implementation that is easily unrolled and structured to reuse values of the $x$ vector.

Our implementation uses the texture cache to load the values of vector $x$, as done by Bell and Garland [3]. We also tried explicit blocking and use of the local-store shared memory but found the texture cache approach to be both simpler and more effective empirically. 


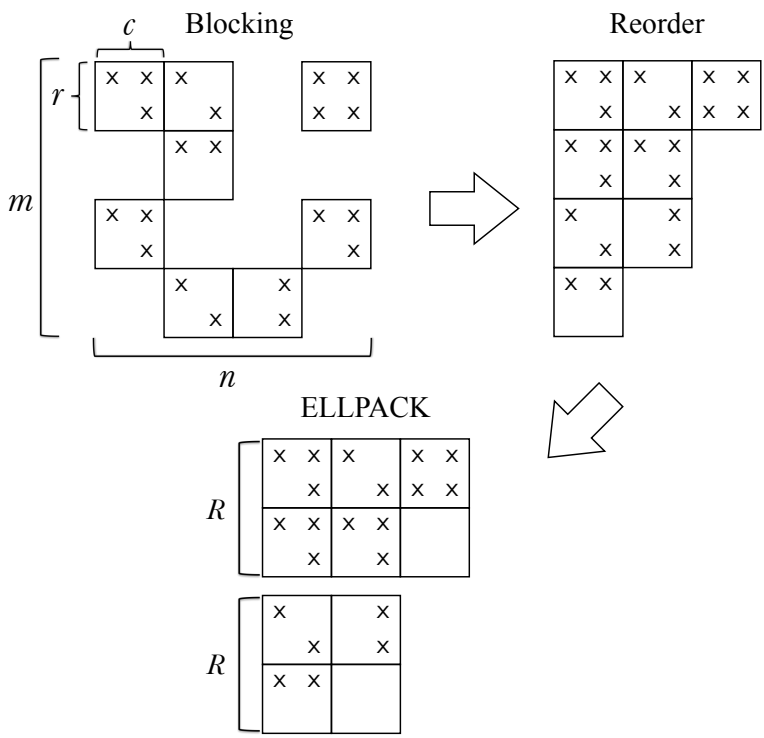

(a) Construction.

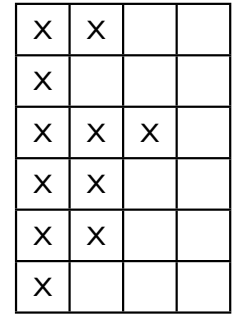

A

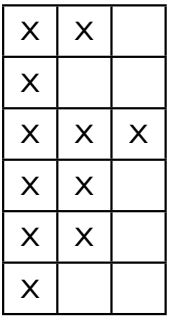

ELLPACK padding $=7$

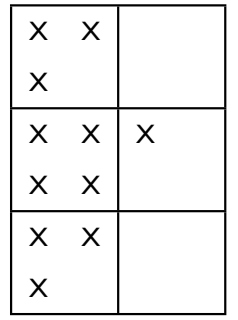

BELLPACK padding $=13$ (b) Severity of padding.

Figure 3. BELLPACK format.

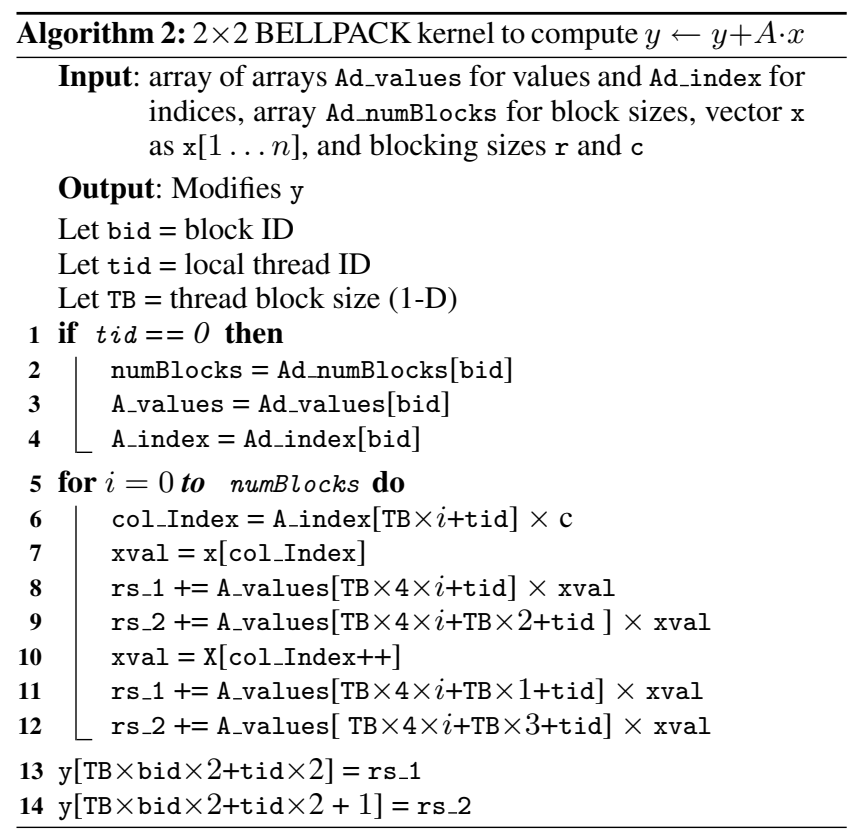

\subsection{Experimental Results}

The performance results for BELLPACK format appear in Figures 5(a) (Gflop/s for single- and double-precision) and 5(b) (estimated effective GB/s in single-precision). We show results on just the subset of matrices which actually have dense block substructure; for the remainder, we would expect the best NVIDIA implementation to deliver the best results.

By "estimated effective bandwidth," we mean the best performance one would expect given (a) an effective sustainable bandwidth as given by the CUDA SDK bandwidthTest benchmark, shown in Table 1; and (b) an optimistic flop:byte ratio of 2 flops for every 4 bytes read, which accounts only for the minimum memory traffic required just to transfer the matrix values (i.e., assuming the "maximum index compression" possible). For example, on the
NVIDIA C1060, we observe a bandwidthTest measurement of 72 $\mathrm{GB} / \mathrm{s}$, which corresponds to an estimated empirical peak performance of $(72 \mathrm{~GB} / \mathrm{s}) \times(2$ flops $/ 4$ bytes $)=36 \mathrm{Gflop} / \mathrm{s}$.

Figure 5 show the best performance found by exhaustively searching through 11 different blocking sizes and 4 different thread block sizes and choosing the best results. Similarly, the "NVIDIA Best" implementation is that of Bell and Garland, showing the best result that we observed over all of the formats available in their implementation [3]. Our BELLPACK implementation is between $1.3 \times$ to $1.8 \times$ faster for single-precision, and between $1.1 \times$ to $1.5 \times$ faster for double-precision, achieving a bandwidth utilization that approaches the peak measured bandwidth.

\section{Model-based Autotuning}

Achieving the best performance from BELLPACK requires carefully tuning the block size parameters, $r, c$, and $R$. These parameters are matrix-dependent, meaning we may need to choose them at run-time. In the spirit of our prior work, we select these parameters using an autotuning framework based on empirical modeling $[11,19,22]$. This model combines off-line benchmarking and a run-time model instantiated with the benchmark data. In contrast to this prior SpMV autotuning work, our model attempts to more directly model GPU hardware features, rather than abstracting the hardware away completely $[11,19]$.

\subsection{A Generic Model of GPU Execution Time}

Our model is based on a simple abstraction of an NVIDIA GPU architecture and its execution model, illustrated in Figure 6.

Basic model: Iterations. We consider a GPU as consisting of $M$ identical streaming multiprocessors (SMs) that execute in parallel. These SMs, which use simultaneous multithreading, can at a given instant in time hold and context-switch among at most $T$ thread blocks. Importantly, $T$ depends on characteristics of both the hardware and the compute kernel (e.g., via the so-called occupancy); we discuss how to compute $T$ in Section 6.2 below.

We model the GPU's execution as all SMs executing its thread blocks in iterations. During each iteration, an individual SM executes up to $T$ thread blocks that it has been assigned. 


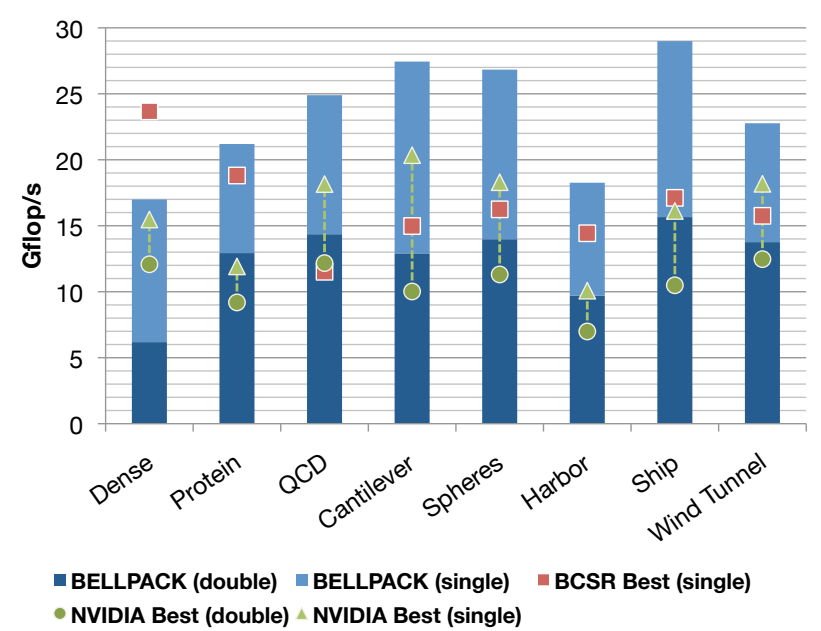

(a) Performance on "naturally blocked" matrices

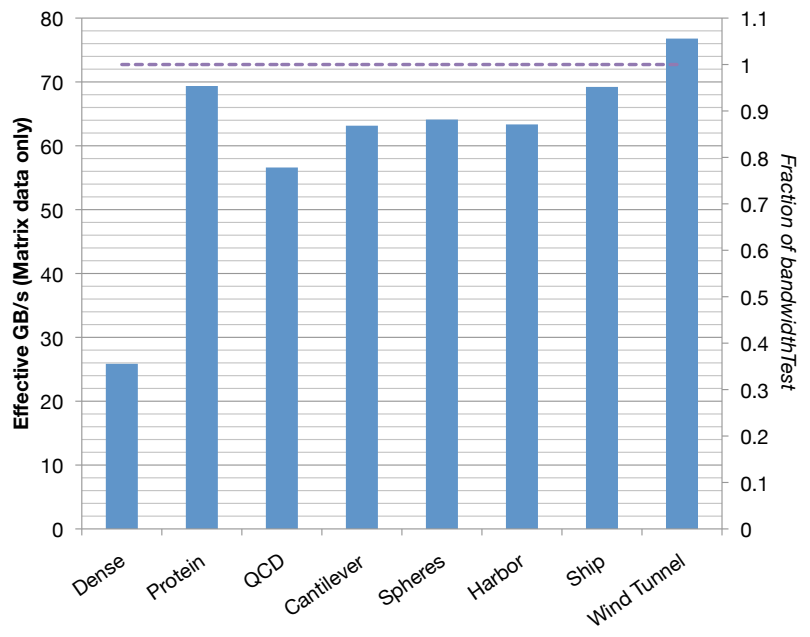

(b) Effective bandwidth (single-precision)

Figure 5. BELLPACK performance on NVIDIA C1060 (T10P multiprocessor)

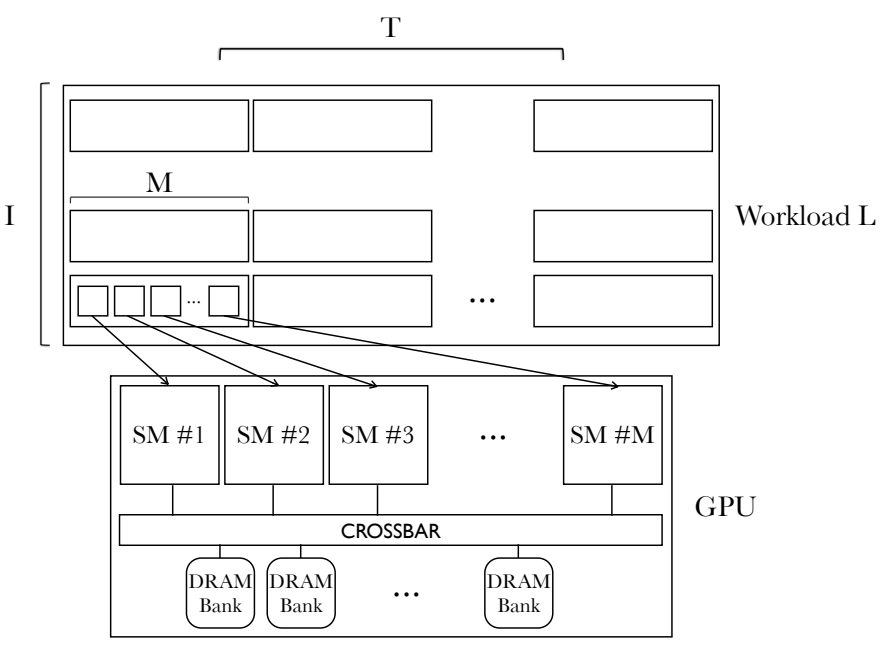

Figure 6. A diagram of NVIDIA GPU architecture and execution model

If the computational workload consists of a total of $L$ thread blocks, we can compute the total number of iterations $I$ under some assumptions. Let us assume that the SMs are "perfectly synchronized" in the sense that, during a given iteration, all SMs on thread blocks consisting of identical work can proceed in close unison. Then, during each iteration, all $M$ SMs will execute up to $T$ thread blocks each, meaning the total number of such iterations is

$$
I=\left\lceil\frac{L}{T \times M}\right\rceil \text {. }
$$

We consider the total execution time to be the sum of the execution times of the individual iterations. Under the assumption of roughly identical work during an iteration, the total time $t$ is

$$
t=\sum_{i=1}^{I} t_{i}
$$

where $t_{i}$ is the time of each iteration. We model this iteration time using a simple linear function of the maximum number of thread blocks, $k$, assigned to any SM during iteration $i$ as follows:

$$
t_{i}(k)=\sigma_{i}+\alpha_{i} \times(k-1),
$$

where $1 \leq k \leq T$; $\sigma_{i}$ models the iteration's startup time, which includes some overhead plus the time to compute the first iteration; and $\alpha_{i}$ measures the degree to which the SM can hide the latency of each thread block through context switching. The first iteration's startup cost, $\sigma_{1}$, could include things like the kernel startup time. Regarding the latency hiding, the smaller the value of $\alpha_{i}$, the better the SM is able to hide the thread block latency.

Homogeneous execution. For the kernels we consider in this paper, we make an additional simplification. Suppose that the thread blocks are largely homogeneous in the sense that $\alpha_{i}=\alpha$ is the same for all iterations and that only the first iteration may have a different overhead term, i.e., $\sigma_{2}=\sigma_{3}=\cdots=\sigma_{I}=\sigma$ but $\sigma_{1}$ may or may not equal $\sigma$. Then, assuming $I \geq 2$, we can write the total time in three terms, namely, the time $\tau_{1}$ for the first iteration, the time $\tau$ for each of the middle $I-2$ iterations, and the time $\tau_{I}$ for the last iteration:

$$
\begin{aligned}
t & =\tau_{1}+(I-2) \times \tau+\tau_{I} \\
\tau_{1} & =\sigma_{1}+\alpha \times(T-1) \\
\tau & =\sigma+\alpha \times(T-1) \\
\tau_{I} & =\sigma+\alpha \times\left\lfloor\frac{(L \bmod (T \times M))-1}{M}\right\rfloor .
\end{aligned}
$$

Comment: Active warps. One last important factor to consider on a GPU is the number of active warps in a single thread block. All operations are done in the granularity of warps, including the interleaving of thread blocks to hide memory latency. If there is only 1 thread block available for each SM and if there are not enough warps in the thread block for latency-hiding via multithreading, then there will be a slight skew in the curve for $t_{i}$ (or $\tau_{i}$ ). That is, the cost of executing the first thread block in an iteration could be much larger than the cost of computing the subsequent thread block. This skew will depend on kernel features such as the number memory operations and the number of warps in a single thread block and hardware factors such as the bandwidth and the number of banks in the memory system [10]. We illustrate this phenomenon in Figure 7. 
1 Thread Block 2 Thread Block 4 Thread Block
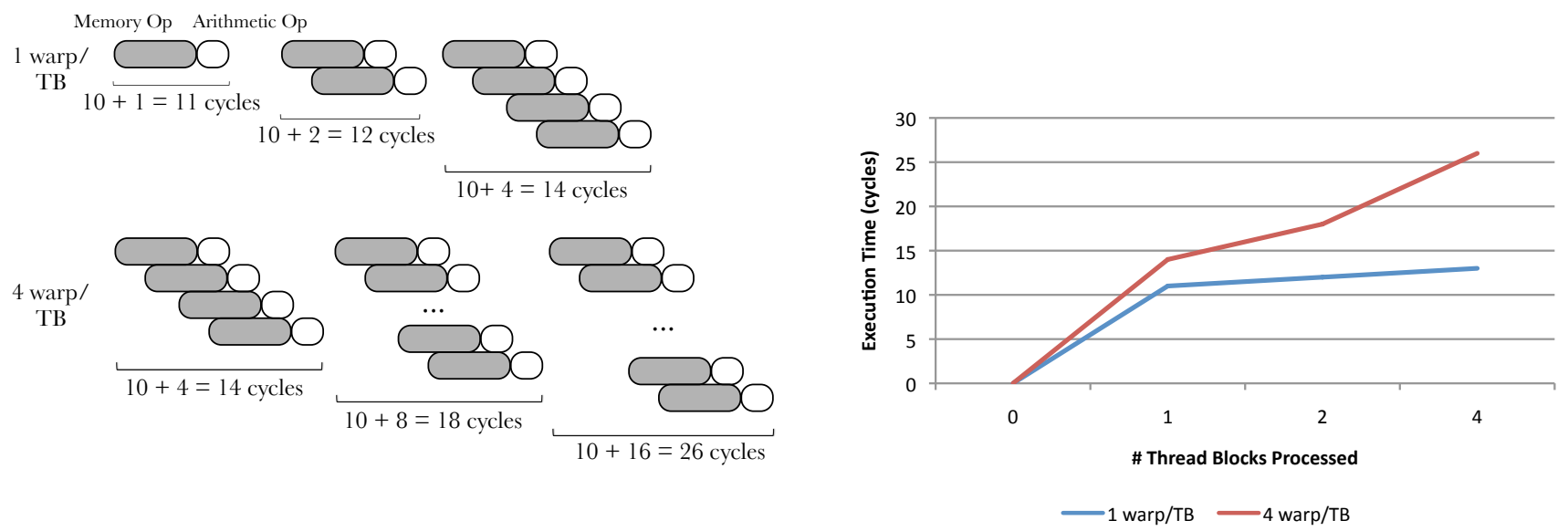

Figure 7. Effect of \# warps per thread block on $t_{I}$

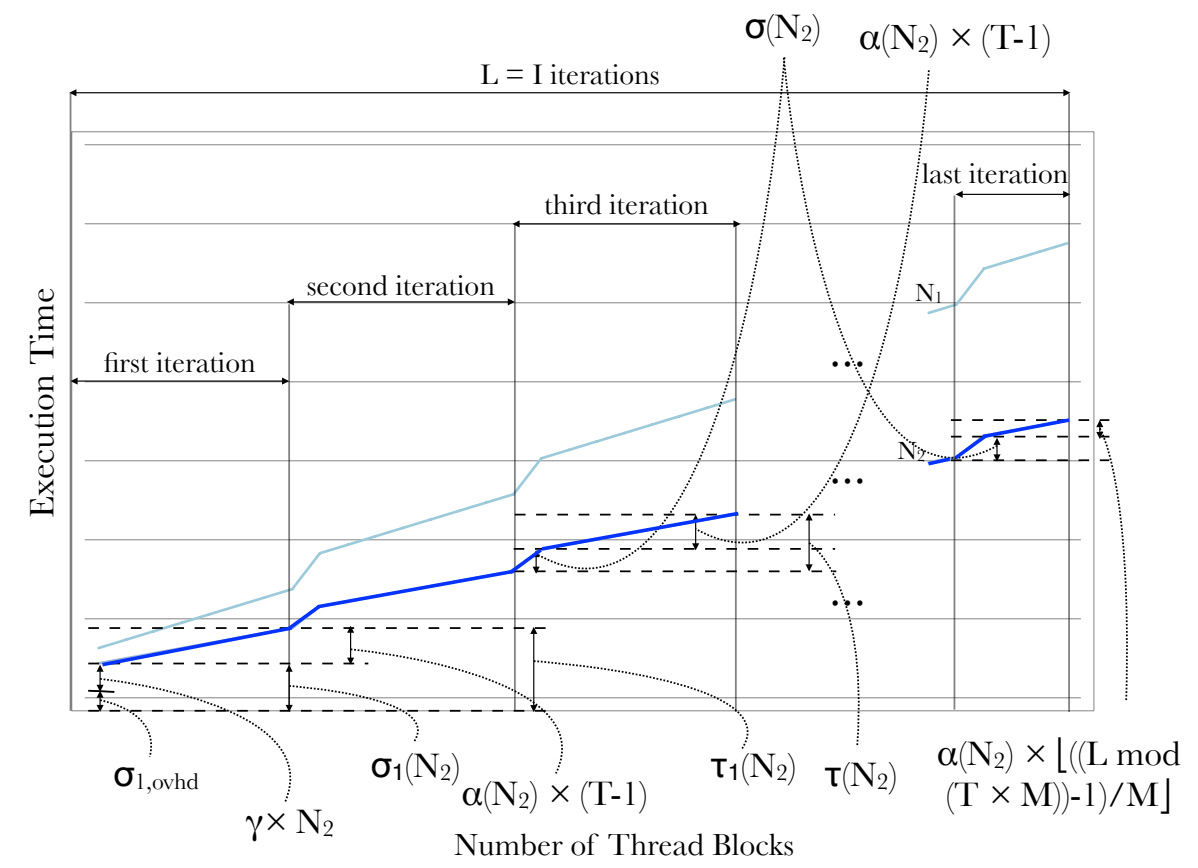

Figure 8. Execution Model Graph

Illustration of the model. The overall execution model described in this section can be summarized graphically, as shown in Figure 8.

\subsection{Computing $T$ (Max. Thread Blocks per Multiprocessor)}

The execution time model of Section 6.1 assumes that at most $T$ thread blocks (TBs) can co-exist on the same SM. This value, which is related to the CUDA's kernel occupancy [1], can be computed for a given GPU and kernel using Equations 8 through 12 below. These equations depend on a number of GPU- and kernel-dependent parameters, which are summarized in Table 3.

$$
\begin{aligned}
W_{T B} & =\frac{K}{W} \\
T_{W} & =\min \left(T_{S M},\left\lfloor\frac{W_{S M}}{W_{T B}}\right\rfloor\right) \\
T_{R} & =\left\lfloor\frac{R_{S M}}{R_{T B}}\right\rfloor \\
T_{S} & =\left\lfloor\frac{S_{S M}}{S_{T B}}\right\rfloor \\
T & =\min \left(T_{W}, T_{R}, T_{S}\right)
\end{aligned}
$$




\begin{tabular}{|c|l|c|}
\hline $\begin{array}{c}\text { Variable } \\
\text { Name }\end{array}$ & Description & Dependence \\
\hline $\mathrm{W}$ & warp size & GPU \\
\hline $\mathrm{K}$ & \# threads per TB & kernel \\
\hline $\mathrm{W}_{S M}$ & maximum \# warps per SM & GPU \\
\hline $\mathrm{W}_{T B}$ & \# warps per TB & Both \\
\hline $\mathrm{T}_{W}$ & $\begin{array}{l}\text { \# thread blocks limited } \\
\text { by warps }\end{array}$ & Both \\
\hline $\mathrm{T}_{S M}$ & max \# thread blocks per SM & GPU \\
\hline $\mathrm{R}_{S M}$ & max \# registers per SM & GPU \\
\hline $\mathrm{R}_{T B}$ & \# register usage per TB & kernel \\
\hline $\mathrm{T}_{R}$ & $\begin{array}{l}\text { \# thread blocks limited } \\
\text { by registers }\end{array}$ & Both \\
\hline $\mathrm{S}_{S M}$ & max shared mem per SM & GPU \\
\hline $\mathrm{S}_{T B}$ & shared mem usage per TB & kernel \\
\hline $\mathrm{T}_{S}$ & $\begin{array}{l}\text { \# thread blocks limited } \\
\text { by shared mem }\end{array}$ & Both \\
\hline
\end{tabular}

Table 3. Table of variables used to calculate $T$

\subsection{Instantiating the Model for SpMV}

To use the model for autotuning SpMV, we need to instantiate an SpMV-specific model. Like related work, we use offline benchmarking [11, 19], but in contrast, we use this data to determine various parameters in the model (e.g., $\sigma, \alpha$ ). In this section, we instantiate a model for the BELLPACK kernel, using the homogenous model (Section 6.1).

Computing the startup cost, $\sigma_{i}$. All of the BELLPACK kernels (one for each value of $r$ and $c$ ) have the same structures. For instance, the kernels all load thread block related data such as the thread and block ID; index into the main data structures; and declare variables. The main portion of the kernel loops over all the $r \times c$ blocks that the thread block is assigned and computes the necessary values. The kernel ends by copying the accumulated values back to the vector $Y$.

The first iteration's startup cost, $\sigma_{1}$, is mainly determined by the number of $r \times c$ blocks it reads. For a $r \times c$ kernel of thread block size $R$, where each thread processes $N$ blocks of size $r \times c$ each, $\sigma_{1}$ and $\sigma$ can be approximated as follows:

$$
\begin{aligned}
\sigma_{1}(N) & =\sigma_{1, \mathrm{o}}+\gamma \times N \\
\sigma(N) & =\gamma^{\prime} \times N .
\end{aligned}
$$

Observe that Equation 13 further decomposes the $\sigma_{1}$ startup into a "base" overhead, $\sigma_{1, \mathrm{o}}$ plus the time $\gamma \times N$ to process the $N$ blocks.

We determine the various factors in Equations 13-14 using offline benchmarks on dense matrices. Specifically, we first estimate $\sigma_{1, \mathrm{o}}$ by measuring the time to execute the kernel on a $(M \times R \times r)$ by- $c$ dense matrix stored in BELLPACK format. We then execute the kernel on a $(M \times R \times r)$-by- $(c \times \beta)$ dense matrix, for some value of $\beta$ (below), and measure its execution time, $\phi_{M \cdot R \cdot r \times c \cdot \beta}$. From these data, we determine $\gamma$ via

$$
\gamma=\frac{\left(\phi_{M \cdot R \cdot r \times c \cdot \beta}-\sigma_{1, \mathrm{o}}\right)}{\beta} .
$$

We compute the subsequent iteration times, $\gamma^{\prime}$, as follows:

$$
\gamma^{\prime}=\frac{\left(\phi_{M \cdot(T+1) \cdot R \cdot r \times c \cdot \beta}-\phi_{M \cdot T \cdot R \cdot r \times c \cdot \beta}\right)}{\beta}
$$

The value $\beta$ can be chosen arbitrarily, but should be kept small ( $\leq$ 100).

Estimating the latency-hiding factor, $\alpha$. The variable $\alpha$ is used to estimate the increase in the execution time as additional thread blocks are added to the SM, and its value reflects the ability to hide latency (Section 6.1). We estimate $\alpha$ by

$$
\alpha(N)=\left(\frac{\phi_{M \cdot T \cdot R \cdot r \times c \cdot \beta}-\phi_{M \cdot R \cdot r \times c \cdot \beta}}{(T-1) \times \beta}\right) \times N,
$$

using previously estimated values.

Final execution time model. The preceeding benchmarks are enough to estimate the execution time for a particular $r \times c$ kernel with thread block size $R$ and $N$ BELLPACK blocks as shown below, again assuming $I \geq 2$. Having the equation vary with $N$ creates a more flexible model and estimates the range of errors that may occur due to variance in blocking size estimation, discussed in a subsequent section.

$$
\begin{aligned}
\tau_{1}(N) & =\sigma_{1}(N)+\alpha(N) \times(T-1) \\
\tau(N) & =\sigma(N)+\alpha(N) \times(T-1) \\
\tau_{I}(N) & =\sigma(N)+\alpha(N) \times\left\lfloor\frac{(L \bmod (T \times M))-1}{M}(20)\right. \\
t(N) & =\tau_{1}(N)+(I-2) \tau(N)+\tau_{I}(N)
\end{aligned}
$$

\subsection{Blocking Size Estimation}

As SpMV is a bandwidth limited application, one simple way of eliminating potential blocking sizes is to estimate the total amount of data that would be generated by using those sizes. For example, for the "Protein" matrix, a blocking size of $3 \times 3$ generates $4.9 \mathrm{M}$ data elements on average, approximately $1.13 \times$ more data elements than the original matrix, whereas blocking sizes of $7 \times 7$ and $8 \times 8$ generate $2.1 \times$ and $2.2 \times$ more data respectively. Since $7 \times 7$ and $8 \times 8$ blocking sizes produce significantly more data, they can be safely eliminated from consideration as long as they can be roughly estimated.

The total data size can be estimated simply randomly sampling a fraction of the matrix and interpolating the results for the rest of the matrix, or by using a fill ratio estimation algorithm [19], that can relatively accurately measure the ratio of number of stored values to the number of non-zeros by sampling only $1 \%$ of the matrix.

\subsection{Autotuning Framework}

Once a list of potential optimal blocking sizes has been created, we can use the equations described in the previous sections to compute the models for the different blocking sizes and thread block size R. A flowchart showing the process is shown in Figure 9.

The framework is flexible as many of these parameters can be fine-tuned to suit the needs of the application. For example, the size of the list of potential blocking sizes can be controlled by tightening the range of total data size allowed, and if certain thread block sizes result in consistently poor performance numbers, those can skipped.

\subsection{Experimental Results}

Dense matrix in sparse format. As partial validation of the model, we compare the model predictions against measurements for a dense matrix in BELLPACK format, shown in Figures 10-12. These measurements are taken on two different GPUs with very different hardware specs to verify that the functional form of the model was not specifically tied to either one of the GPUs.

The model estimates the performance closely with average and median error of $3.74 \%$ and $2.73 \%$ respectively, and maximum error of $14.89 \%$ for the $1 \times 2$ kernel for $R=32$. The $3 \times 3$ kernel for $R=128$ has higher average and median error of $13.56 \%$ and $2.95 \%$ respectively, and maximum error of $61.41 \%$. This higher value of average error but low median error value for the $3 \times 3 \mathrm{kernel}$ is due to a particular value of $N$ having consistently large error $(\geq 60 \%)$. 

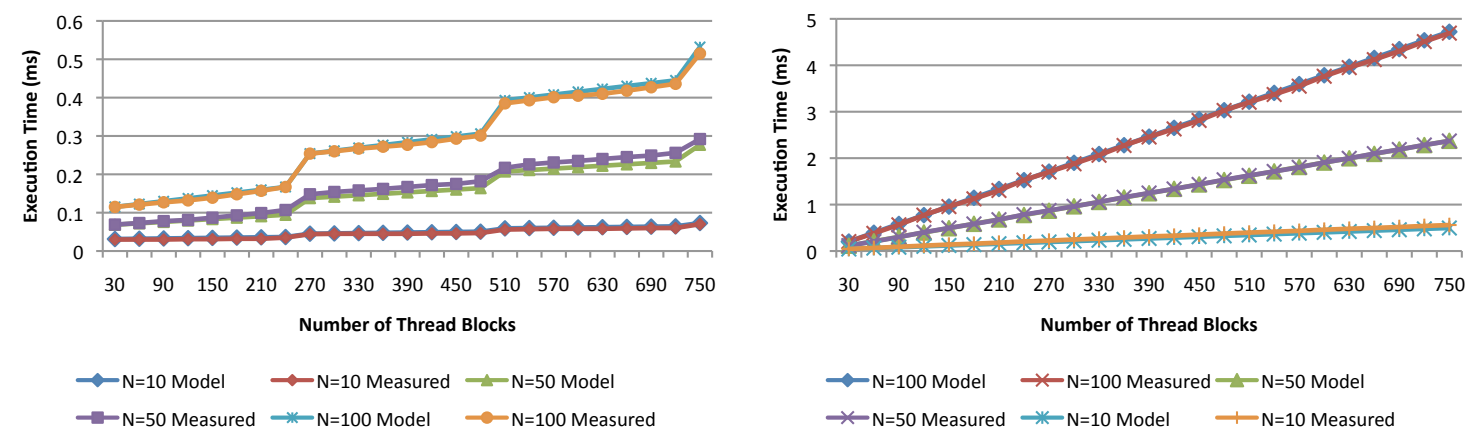

Figure 10. Comparison of model to real data for BELLPACK on T10

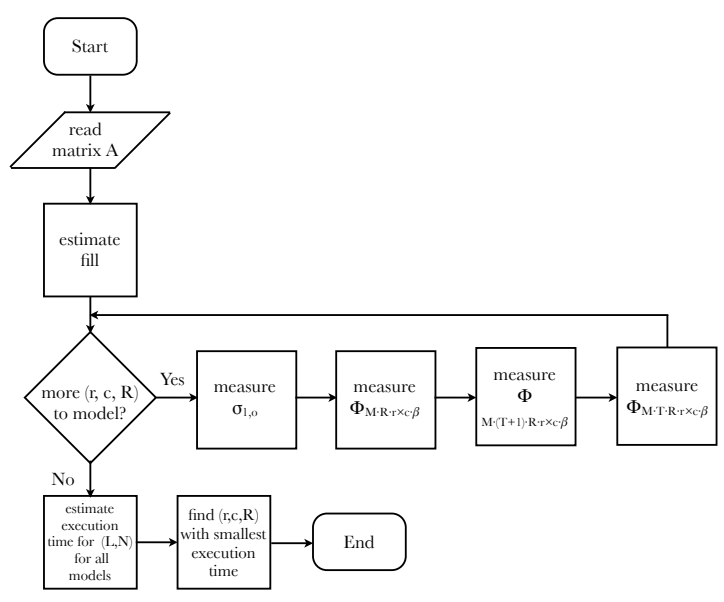

Figure 9. Autotuning Framework

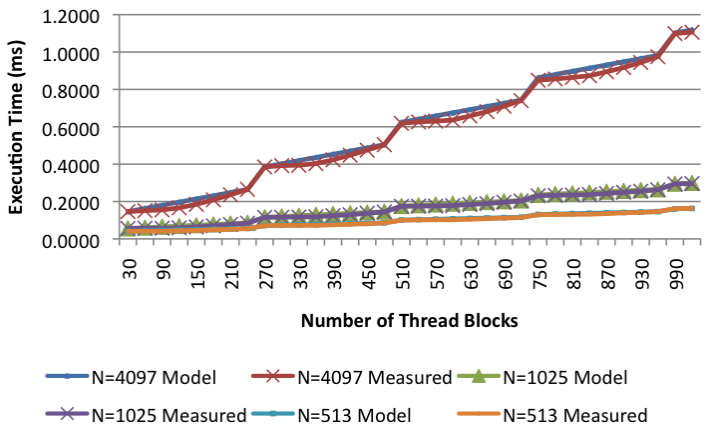

Figure 11. Comparison of model to real data for BCSR on T10

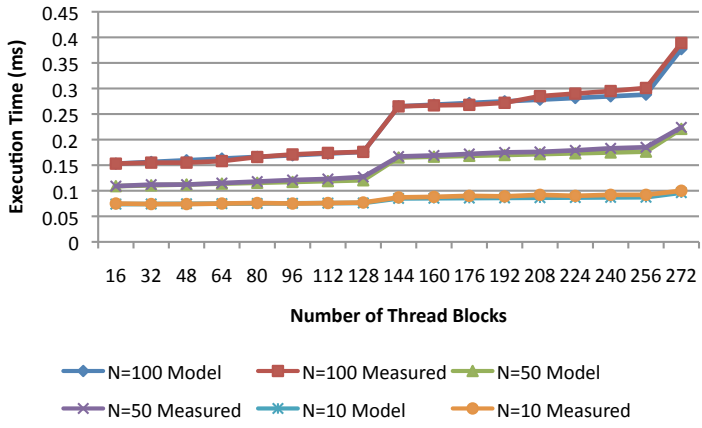

Figure 12. Comparison of model to real data for BELLPACK on C870

The same model was tested on a Tesla C870 also, which has a different number of SM and hardware resources. Figure 12 shows that the same performance model works across different hardware. The average and median error rates were $2.18 \%$ and $2.06 \%$ respectively, and the maximum error was $6.18 \%$.

Tests on the matrix suite. The results obtained by autotuning as compared to the best performance found by exhaustive search is shown in Figure 13. Since BELLPACK is designed only for matrices with dense block substructure, we evaluate only against those 8 matrices. Such structure can be quickly and easily detected [19]. Figure 13 shows that we find near-optimal block sizes in 5 of 8 cases, with the non-optimally tuned kernels achieving $86.5 \%$ of the best performance numbers on average (median of $92.6 \%$ ).

The autotuning was done over $1 \times 2,2 \times 2,2 \times 3,3 \times 2,3 \times 3,3 \times 4$, $4 \times 4,5 \times 5,6 \times 6,7 \times 7$, and $8 \times 8$ kernels, for $R$ sizes of $32,64,128$, and 256 each, a total of 44 possible combinations. Some blocking sizes such as $7 \times 7$ and $8 \times 8$ were eliminated by fill estimation for most matrices, as they yielded extremely large data sizes.

An $86.5 \%$ average accuracy $(92.6 \%$ median) relative to exhaustive search leaves room for improvement. Nevertheless, even for the worst mispredictions, the performance of the selected optimization is still at least as fast as the best NVIDIA implementation.

\subsection{Alternatives to our model-based approach}

There are a number of other approaches. Exhaustive search is one example. However, the relatively high cost of transforming the data structure (e.g., up to $40 \mathrm{SpMVs} \mathrm{[19])} \mathrm{rules} \mathrm{out} \mathrm{this} \mathrm{technique} \mathrm{for}$ use at run-time. A second example is a memory footprint minimization heuristic. Since SpMV is memory bandwidth-bound and, to first order, limited by the time to stream the matrix, one might 


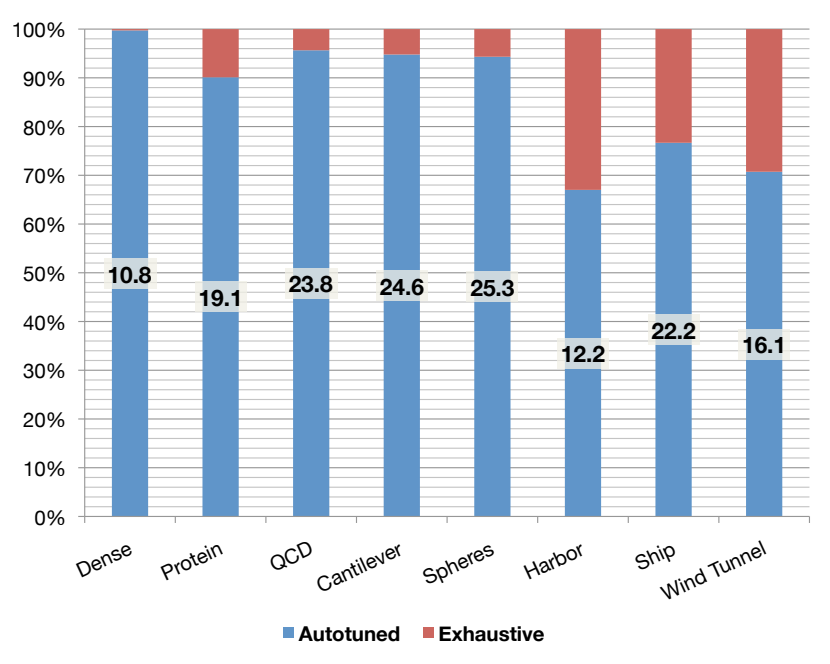

Figure 13. Performance of the implementation found using our autotuning model, as a fraction of the performance of the implementation found by exhaustive search. The numerical values on each bar indicate the Gflop/s of the autotuned implementation.

reasonably expect the optimal block size to be the one that minimizes the matrix data structure footprint. However, in our exhaustive search-based experiments, we found this heuristic to yield mixed results. The main cause is is that this heuristic ignores the actual kernel performance, since each data structure we consider has an associated kernel computation, and that kernel performance can vary widely and in unintuitive ways depending on register allocation, alignment, and other issues.

\section{Conclusions and Future Work}

Our findings lend further support to the intuition that a memory bandwidth-rich GPU platform can deliver excellent absolute performance on SpMV, at least for the class of matrices with dense block substructure, such as those arising in finite element method applications. The absolute performance numbers we achieve are among the best published thus far across a wide spectrum of singlenode multisocket multicore platforms, including prior GPU SpMV results [2,3] and the STI Cell Broadband Engine [21].

To make our BELLPACK-based SpMV practical, however, requires a judicious choice of data structure tuning parameters, a problem not addressed in prior GPU SpMV work. We contribute a GPU-specific execution time model, inspired by the general CUDA programming model, that can accurately predict suitable tuning parameters. The main limitation of our study is that we have thus far only validated it with respect to our BELLPACK and BCSR SpMV implementations on GPUs. However, as part of our on-going work, we continue to validate and refine the model on additional kernels. We believe that with the appropriate level of additional refinement, we could provide insight into the performance of more general GPU-like architectures, providing both a way to "tune" an architecture via modeling as well as providing an analytical modeling tool for designing algorithms and their implementations.

\section{Acknowledgments}

This work was supported in part by the National Science Foundation (NSF) under award number 0833136, NSF TeraGrid allocation CCR-090024, joint NSF 0903447 / Semiconductor Research Corporation (SRC) Award 1981, and a grant from the Defense Advanced Research Projects Agency (DARPA). Any opinions, find- ings and conclusions or recommendations expressed in this material are those of the authors and do not necessarily reflect those of NSF, SRC, or DARPA.

\section{References}

[1] NVIDIA CUDA (Compute Unified Device Architecture): Programming Guide, Version 2.1, December 2008.

[2] Muthu Manikandan Baskaran and Rajesh Bordawekar. Optimizing sparse matrix-vector multiplication on GPUs using compile-time and run-time strategies. Technical Report RC24704 (W0812-047), IBM T.J. Watson Research Center, Yorktown Heights, NY, USA, December 2008.

[3] Nathan Bell and Michael Garland. Efficient sparse matrix-vector multiplication on CUDA. In Proc. ACM/IEEE Conf. Supercomputing (SC), Portland, OR, USA, November 2009. (to appear).

[4] Jeff Bolz, Ian Farmer, Eitan Grinspun, and Peter Schröder. Sparse matrix solvers on the GPU: Conjugate gradients and multigrid. In Proc. Special Interest Group on Graphics Conf. (SIGGRAPH), San Diego, CA, USA, July 2003. doi: http://dx.doi.org/10.1145/882262.882364.

[5] Matthias Christen and Olaf Schenk. Genera-purpose sparse matrix building blocks using the NVIDIA CUDA technology platform. In Proc. Workshop on General-Purpose Processing on Graphics Processing Units (GPGPU), Boston, MA, USA, October 2007.

[6] Eduardo F. D'Azevedo, Mark R. Fahey, and Richard T. Mills. Vectorized sparse matrix multiply for compressed row storage. In Proc. Int'l. Conf. Computational Science (ICCS), volume 3514/2005 of LNCS, pages 99-106. Springer Berlin / Heidelberg, 2005. doi: http://dx.doi.org/10.1007/11428831_13.

[7] James Demmel, Jack Dongarra, Viktor Eijkhout, Erika Fuentes, Antoine Petitet, Richard Vuduc, R. Clint Whaley, and Katherine Yelick. Self-adapting linear algebra algorithms and software. Proc. IEEE, 93(2):293-312, February 2005. doi: http://dx.doi.org/10.1109/JPROC.2004.840848.

[8] Michael Garland. Sparse matrix computations on manycore GPUs. In Proc. ACM/IEEE Design Automation Conf. (DAC), pages 2-6, Anaheim, CA, USA, 2008. doi: http://dx.doi.org/10.1145/1391469.1391473.

[9] Roman Geus and Stefan Röllin. Towards a fast sparse symmetric matrix-vector multiplication. Parallel Computing, 27(7):883-896, June 2001. doi: http://dx.doi.org/10.1016/S0167-8191(01)00073-4.

[10] Sunpyo Hong and Hyesoon Kim. An analytical model for a GPU architecture with memory-level and thread-level parallelism awareness. In Proc. ACM Int'l. Symp. Comp. Arch. (ISCA), pages 152-163, Austin, TX, USA, June 2009. doi: http://dx.doi.org/10.1145/1555815.1555775.

[11] Eun-Jin Im, Katherine Yelick, and Richard Vuduc. SPARSITY: Optimization framework for sparse matrix kernels. Int'l J. of High Performance Computing Applications (IJHPCA), 18(1):135-158, February 2004. doi: http://dx.doi.org/10.1177/1094342004041296.

[12] Hiroshi Okuda, Kengo Nakajima, Mikio Iizuka, Li Chen, and Hisashi Nakamura. Parallel finite element analysis platform for the Earth Simulator: GeoFEM. In Proc. Int'l. Conf. Computational Science (ICCS), volume 2659 of $L N C S$, pages 773-780. Springer, 2003. doi: http://dx.doi.org/10.1007/3-540-44863-2_75.

[13] Ali Pinar and Michael T. Heath. Improving performance of sparse matrix-vector multiplication. In Proc. ACM/IEEE Conf. Supercomputing (SC), Portland, OR, USA, 1999. doi: http://dx.doi.org/10.1145/331532.331562.

[14] John R. Rice and Ronald F. Boisvert. Solving elliptic problems using ELLPACK. Springer Verlag, 1984.

[15] Yousef Saad. SPARSKIT: A basic tool kit for sparse matrix computations, version 2 . http://www-users.cs.umn.edu/ saad/software/SPARSKIT /sparskit.html, March 2005.

[16] Shubhabrata Sengupta, Mark Harris, Yao Zhang, and John D. Owens. Scan primitives for GPU computing. In Proc. ACM 
SIGGRAPH/EUROGRAPHICS Symp. Graphics Hardware, San Diego, CA, USA, 2007.

[17] Vasily Volkov and James W. Demmel. Benchmarking GPUs to tune dense linear algebra. In Proc. ACM/IEEE Conf. on Supercomputing (SC), Austin, TX, USA, November 2008.

[18] Richard Vuduc, James W. Demmel, and Katherine A. Yelick. OSKI: A library of automatically tuned sparse matrix kernels. In Proc. SciDAC, J. Phys.: Conf. Series, volume 16, pages 521-530, 2005. doi: http://dx.doi.org/10.1088/1742-6596/16/1/071.

[19] Richard W. Vuduc. Automatic performance tuning of sparse matrix kernels. PhD thesis, University of California, Berkeley, CA, USA, January 2004.
[20] Richard W. Vuduc and Hyun-Jin Moon. Fast sparse matrix-vector multiplication by exploiting variable block structure. In Proc. HighPerformance Computing and Communications Conf., volume LNCS 3726/2005, pages 807-816, Sorrento, Italy, September 2005. Springer. doi: http://dx.doi.org/10.1007/11557654_91.

[21] Sam Williams, Richard Vuduc, Leonid Oliker, John Shalf, Katherine Yelick, and James Demmel. Optimizing sparse matrix-vector multiply on emerging multicore platforms. Journal of Parallel Computing, 35(3):178-194, March 2009. doi: http://dx.doi.org/10.1016/j.parco.2008.12.006.

[22] Kamen Yotov, Xiaoming Li, Gang Ren, María Jesús Garzarán, David Padua, Keshav Pingali, and Paul Stodghill. Is search really necessary to generate high-performance BLAS? Proc. IEEE, 93(2):358-386, February 2005. doi: http://dx.doi.org/10.1109/JPROC.2004.840444. 